\title{
Rechthaben müssen oder leben wollen: Über Martin Walsers Muttersohn
}

\section{Silke Horstkotte}

Im Vorfeld der deutschen Bundestagswahl fragte die Zeit in ihrer Ausgabe vom 5. September 2013 achtundvierzig "namhafte Künstler und Intellektuelle«, welche Partei sie wählen würden. An erster Stelle durfte Martin Walser antworten und gab das folgende bemerkenswerte Statement zu Protokoll:

"Herr Steinbrück will mit jedem Satz einen Treffer landen. Frau Merkels Sätze klingen nicht so, als wären sie schon fertig gewesen, bevor sie gesagt wurden. Frau Merkel musste ihre Sätze suchen, und wir atmeten auf, wenn sie sie fand. Die Sätze von Herrn Steinbrück wirkten im TV-Duell oft gebraucht. Herr Steinbrück versuchte zu verbergen, wie überlegen er sich fühlte. Er wusste viel mehr Zahlen als Frau Merkel. Frau Merkel wirkte mehr durch sich selbst. Zahlen kann ich nicht nachprüfen. Dasein ist erlebbar. Frau Merkel musste nur da sein. Wer recht haben muss, muss Herrn Steinbrück wählen. Wer leben will, kann Frau Merkel wählen. Und ich habe schon vor mehr als zehn Jahren eingesehen und formuliert, dass das Rechthaben kein nennenswerter Bewusstseinszustand ist. "1

Damit wäre nicht nur die vieldiskutierte Frage endgültig entschieden, ob es überhaupt Wähler gibt, die sich durch TV-Duelle beeinflussen lassen - und das oft gehörte Gegenargument entkräftet, es seien doch gar nicht Personen, sondern Parteien, die in Deutschland zur Wahl stehen. Nein, Walsers im unverkennbaren Walser-Sound vorgetragene Wahlrechtfertigung verbindet sich auch, geradezu programmatisch, in zweifacher Weise mit seinem literarischen und essayistischen Werk. Nämlich einmal mit der poetologischen Frage nach dem richtigen Reden und der gelungenen Kommunikation, die zahlreiche Walserfiguren umtreibt, und zum anderen mit der theologisch-anthropologischen Frage nach der Rechtfertigung und dem Gerechtfertigtsein in seinem Gegensatz zum Rechthaben, die im Mittelpunkt des Essays Über Rechtfertigung steht. ${ }^{2}$

1 Die Qual der Wahl, in: Die Zeit, 5.9. 2013.

2 Auch in Über Rechtfertigung werden Rechthaben und Gerechtfertigtsein als rhetorische Qualitäten plakativ an der Antithese zweier Redner festgemacht, nämlich 
In provokanter Weise begründet Walser seine Wahlentscheidung nicht etwa durch die Wahlprogramme der beiden größten deutschen Parteien und deren Verhältnis zu seinen eigenen politischen Überzeugungen; tatsächlich spielen genuin politische Erwägungen in seiner Argumentation keine Rolle. (Auch seine Mitkommentatoren im Zeit-Feuilleton zeigten sich bemerkenswert unbekümmert durch politische Überlegungen.) Vielmehr macht Walser seine Wahl von der rhetorischen Ausgestaltung der Repliken Steinbrücks und Merkels, von actio und persuasio abhängig, plädiert dabei paradoxerweise aber gerade für ein anti-rhetorisches Suchen nach den Sätzen, für das Unfertige, das er in Merkels Antworten erkannt haben will. Mit diesem Kniff gelingt es ihm, sein Votum für die amtierende Bundeskanzlerin, deren Partei zu diesem Zeitpunkt in allen Wahlumfragen weit vor der SPD lag, als eine Parteinahme für das Einfache und Schwache erscheinen zu lassen. Während zahlreiche Medienanalysen Merkel im Anschluss an das TV-Duell einen routinierten »Panzer von freundlich-unverbindlichen Sätzen" zuschrieben, ${ }^{3}$ an dem Steinbrück "zerschellt" sei, ${ }^{4}$ sieht Martin Walser in Angela Merkel ein gerade nicht gekünstelt-routiniertes, sondern reines, authentisches und für den Zuschauer nacherlebbares Dasein verkörpert. Das eigentlich Bemerkenswerte an Walsers Wahlaussage ist dabei, dass er sie auf einen anthropologischen Grundwiderspruch zurückführt, der eine Entscheidungsgrundlage nicht nur für Martin Walser, sondern in der Tat für alle Wählerinnen und Wähler liefert: "Wer recht haben muss, muss Herrn Steinbrück wählen. Wer leben will, kann Frau Merkel wählen.«

Dass Martin Walser sich hier einmal mehr als enfant terrible des Literaturbetriebs aufzuspielen versucht: geschenkt. Dass er mit dem Gegensatz von aufgesetztem und antrainiertem Rechthaben (müssen) einerseits, echtem, authentischem und einfachem Leben (können) andererseits eine nicht nur poetisch-rhetorische, sondern auch theologische Antithese formuliert - nämlich die von Zwang

Jean Ziegler und Joachim Gauck. M. Walser, Über Rechtfertigung, eine Versuchung. Reinbek 2012, 13-17.

3 R. Pörtner, Steinbrück misslingt der Durchbruch, in: Stuttgarter Zeitung, 2.9. 2013 $<$ http://www.stuttgarter-zeitung.de/inhalt.kommentar-zum-tv-duell-steinbrueckmisslingt-der-durchbruch.fb674b52-b7b5-4a59-b938-8534e6dbcea2.html> (eingesehen am 8.9.2013).

4 So der Kommentar der Stuttgarter Zeitung; vgl. die Presseschauen in der Zeit <http:// www.zeit.de/politik/deutschland/2013-09/tv-duell-merkel-steinbrueck-presse> und in der Welt <http://www.welt.de/politik/deutschland/article119611346/AmGelassenheits-Bollwerk-der-Kanzlerin-zerschellt.html> (beide eingesehen am 8.9. 2013). 
und Freiheit -, kann jedoch als Spur in den Rechtfertigungskomplex im Werk Walsers dienen. Diese Spur verfolge ich in diesem Aufsatz in einem explizit literaturwissenschaftlichen Interesse, nämlich hinsichtlich ihrer fiktionspoetischen Ziele, die am Beispiel des 2011 erschienenen Romans Muttersohn exemplarisch erörtert werden sollen. Die späten Walser-Texte, insbesondere die explizit religionsfixierten wie Muttersohn, Über Rechtfertigung und Das dreizehnte Kapitel, sind von der germanistischen Literaturwissenschaft bisher weitgehend ignoriert worden, haben aber großes Interesse bei Theologen, insbesondere bei katholischen, erregt. ${ }^{5}$ Deren enthusiastische Walser-Rezeption kann ich mir eigentlich nur damit erklären, dass man heilfroh ist, wenn sich überhaupt einmal jemand das Wort "Gott « gönnt ${ }^{6}$ - und dann auch noch über Reliquien redet! Doch haben die Versuche, in Walsers Texten eine predigttaugliche Botschaft zu finden, bisher den Blick auf die literarische Verfasstheit gerade der fiktionalen Texte verstellt. Aber in literarischen Texten wird Bedeutung nun einmal nicht allein durch den Stoff, sondern ebenso durch die von diesem gar nicht zu trennende Gestaltung der erzählerischen Vermittlung generiert. Wenn über Religiöses in fiktionalen Erzähltexten verhandelt wird, so impliziert das immer eine doppelte Frage nach der poetischen Funktionalisierung der Religion einerseits, den religiösen Konsequenzen des Poetischen andererseits. Walser selbst legt diese doppelte Konsequenz nahe, wenn er für das "Lebenwollen" als eine nicht ethisch-religiöse, sondern rhetorische Haltung plädiert. Tatsächlich stehen fiktionspoetische Erwägungen auch im Vordergrund von Über Rechtfertigung.

\section{Über Rechtfertigung und Literatur}

Martin Walser erklärt uns in Über Rechtfertigung ja nicht nur, was Rechtfertigung ist - er macht uns auch vor, wie man sich rechtfertigt: nämlich literarisch. Er hat, sagt Walser, Kafka gelesen und ist darüber zum Schriftsteller geworden. Und weil er das Schreiben

\footnotetext{
5 So enthält der Band Mein Jenseits: Gespräche über Martin Walsers "Mein Jenseits « Beiträge von 15 katholischen Theologen - sowie von dem Schriftsteller Arnold Stadler, der in seiner Jugend ebenfalls katholische Theologie studierte.Vgl. M. Felder (Hg.), Mein Jenseits: Gespräche über Martin Walsers "Mein Jenseits", Berlin 2012; sowie J. Badewien/H. Schmidt-Bergmann (Hg.), Martin Walser: Lebens- und Romanwelten, Karlsruhe 2008; J.-H. Tück (Hg.), Was fehlt, wenn Gott fehlt? Martin Walser über Rechtfertigung - theologische Erwiderungen, Freiburg 2013.

6 So der Titel einer Überblicksdarstellung von G. Langenhorst: »Ich gönne mir das Wort Gott«: Gott und Religion in der Literatur des 21. Jahrhunderts, Freiburg 2009.
} 
von Kafka gelernt hat, muss er ein gerechtfertigter Schriftsteller sein, denn alles, was ihm deutsche Feuilletons seit den sechziger Jahren vorgeworfen haben, kann gar nicht stimmen: er kann kein Kommunist, kein Nationalist und natürlich erst recht kein Antisemit sein. (Hegel und Marx hat er nebenher auch gelesen.)

Was passiert, wenn ein derartig mit allen Wassern gerechtfertigter Schriftsteller einen Roman über den Glauben schreibt? Walser hat in Über Rechtfertigung die Heilsgeschichte und ihre theologische Deutung als Roman gelesen und an poetologischen Kriterien wie Aufbau und Spannung gemessen. ${ }^{7}$ Religion, so Walser, sei "eine Ausdrucksart wie andere, wie Literatur, Musik, Malerei. Ich lese Religion als Literatur. ${ }^{8}$ Noch die Augustinische massa damnataTheologie wird so als Roman lesbar, zu dem dann eben eine "Portion Weltfremdheit" gehöre - »eine außerordentliche Zumutung, [...] also mindestens, dass Gott gerecht ist, obwohl er Gnade ausschüttet, über wen er will, und dass dazu der Mensch nichts, absolut nichts tun kann. In diesem Verhältnis ist eben ausgedrückt, wie ungerecht es zugeht in dieser Welt. $\ll^{9}$ Das aber bedeutet nicht nur, an Religion ästhetische Kriterien anzulegen, wie das etwa Martin Mosebach in seiner Häresie der Formlosigkeit tut. ${ }^{10}$ Die Rezeptionshaltung des "Lesen als« ist nicht die Haltung des Ästhetizisten, die alles der Schönheit der Form unterordnet: sie ist diejenige des Lesers fiktionaler Texte, die das Fiktionale unter dem Vorbehalt des "als ob« in ein Verhältnis zur fiktionsexternen Realität setzt.

Unter dieser Maßgabe sind die explizite Berufung auf die theologischen Kernwörter "Glauben" und "Gnade" und die narrative Auseinandersetzung mit der katholischen Tradition - Klöster, Reliquienverehrung - in Muttersohn, ebenso wie die intertextuelle Aneignung hermetischer Bestände (Böhme, Seuse, Swedenborg), dahingehend $\mathrm{zu}$ hinterfragen, ob hier tatsächlich, wie die vorliegenden theologischen Beiträge annehmen, Glaube in neuer Weise zur Sprache gebracht wird: ${ }^{11}$ das wäre das Schreibprogramm der

\footnotetext{
7 »Wenn hier jemand abschaltet, weil es Gott für ihn nicht gibt, also die Frage, ob Gott gerecht sei, für ihn ein Nullproblem ist, zu dem sage ich vorläufig: Lesen wir's als Roman. Madame Bovary und Iwan Karamasow gibt es auch nicht [...].« (Walser, Über Rechtfertigung, 35)

8 Ebd., 32.

9 Ebd., 42.

${ }^{10}$ M. Mosebach, Häresie der Formlosigkeit: Die römische Liturgie und ihre Feinde [2002/2007], München 2012.

${ }^{11}$ So vor allem K.-J. Kuschel, >Ohne das Geglaubte wäre die Welt immer noch wüst und leer: Martin Walser über Religion, in: Felder (Hg.), Mein Jenseits: Gespräche über Martin Walsers »Mein Jenseits«, 72-83.
} 
postsäkularen Poetik. ${ }^{12}$ Oder werden Glaubensbestände und unterschiedliche Formen religiöser Rede hier als rhetorische Strategie in den Dienst der Romanpoetik gestellt? Das wäre das kunstreligiöse Schreibprogramm.

Natürlich ist Muttersohn ein moderner Jesus-Text mit einem Jesus incognito in der Tradition von Dostojewskys Fürst Myschkin. Walsers rätselhafter Protagonist Percy Anton Schlugen hat keinen Vater; er ist ein unsteter Wanderprediger, der eine unzeitgemäße Botschaft der Liebe und der Heilung verkündet. Damit zieht der in einem psychiatrischen Krankenhaus als Pfleger ausgebildete Percy eine Jüngerschaft aus Kranken, Gescheiterten und oberschwäbischen Pfarrhausköchinnen an, doch wird er von der modernen Mediengesellschaft verspottet, auch funktioniert sein Heilungsprogramm nicht: der Klinikinsasse Ewald Kainz, einer der Wunschväter Percys, bringt sich nach Percys "Behandlung" um. Schließlich wird Percy von der Motorradgang Jollynecks, die sich fanatisch dem Hass verschrieben hat, unschuldig ermordet. Auf dieser Ebene ist Muttersohn sicherlich als ein postsäkularer Text lesbar. Da dieser Pfad, wie gesagt, schon öfter beschritten worden ist, werde ich ihn im folgenden aber nicht weiter verfolgen, höchstens gelegentlich kreuzen.

Andererseits ist Muttersohn nämlich auch ein meta-poietischer Roman, der verschiedene Arten von Mitteilungen in selbstbezüglicher Weise thematisiert und in diesem Vollzug das Gelingen und Misslingen rhetorischer Kommunikation auslotet. Von Percys unvorbereiteten, begeisterten Reden im PLK Scherblingen - einem ehemaligen Prämonstratenser-Kloster - über die poetische Wechselrede seiner Mutter Fini mit deren Verlobtem Hugo Schwillk, die volltrunkenen Arno Schmidt-Rezitationen Schwillks und die politischen Reden Ewald Kainz' auf dem Stuttgarter Schlossplatz bis hin zu den dadaistischen Lyrik- und Tanzdarbietungen des PsychiatriePatienten Friedlein Vogel präsentiert der Roman eine Fülle rhetorischer Fallgeschichten. Diese Modelle dienen in Muttersohn immer wieder dazu, eine latent erotische Poetik wechselseitigen Verstehens $\mathrm{zu}$ postulieren, aber auch zu problematisieren. Hinzu kommt ein zweites: die anhand der Figuren und ihrer fiktionsinternen Kommunikation entwickelte Poetik wird reflektiert und komplementiert in der Form des Romans, die auf einer "postalischen" Übertragung

\footnotetext{
${ }^{12}$ Hierzu S. Horstkotte, Poetische Parusie: Zur Rückkehr der Religion in die Gegenwartsliteratur, in: Zeitschrift für deutsche Philologie, Sonderheft zum Band 131: Deutschsprachige Literatur(en) seit 1989 (2012), 265-282; sowie A. Grözinger/A. Mauz/A. Portmann (Hg.), Religion und Gegenwartsliteratur: Spielarten einer Liaison, Würzburg 2009; Langenhorst, "Ich gönne mir das Wort Gott«.
} 
von Texten und Bekenntnissen, aber auch von symbolhaften Dingen wie der Scherblinger Heilig-Blut-Reliquie und von ganzen Traditionen basiert. ${ }^{13}$ In diese Reihe gehört die Form des Romans selbst, die in vier Teilen zunächst die Erzählung über Percys Mission im PLK Scherblingen (I Dem Leben zuliebe), sodann die an Percy übergebene Lebensbeichte Ewald Kainz' (II Dieses Leben) und die Bekenntnisschrift des Klinikchefs und Reliquiendiebes Prof. Augustin Feinlein (III Mein Jenseits) enthält und die mit dem Bericht über den Umzug Feinleins und Percys von Scherblingen nach Rheinau, den Tod Feinleins und die Ermordung Percys durch die Motorradgang (IV Fortleben) schließt.

Das heißt, dass in die heterodiegetische Erzählung über Percy Schlugen zwei intradiegetisch-autodiegetische Texte eingeschlossen sind, die durch die Weitergabe von Dokumenten an die Erzählung erster Ordnung vermittelt werden. Der zweite dieser Texte, Mein Jenseits, war von Walser bereits vorab mit der Gattungsbezeichnung "Novelle« versehen einzeln publiziert worden - allerdings nicht in Walsers neuem Hausverlag Rowohlt, sondern in der deutlich weniger einschlägigen Berlin University Press. ${ }^{14}$ Dass der intradiegetische Erzähler Feinlein in Mein Jenseits vehement für die Schönheit des Reliquienkults und überhaupt der katholischen Tradition seiner oberschwäbischen Heimat eintritt, die auch diejenige Walsers ist, hat diesen Teil des Romans zu einem Lieblingstext der theologischen Leser gemacht. ${ }^{15}$ Dabei ist jedoch das formkonstitutive Moment der Adressierung und Weitergabe bekenntnishafter Texte übersehen worden, der dem "Jenseits«-Teil seine Stellung innerhalb der Formkomposition des Romans zuweist: Feinleins Bekenntnis ist durchgängig an Percy adressiert und schließt mit dem Gruß»BIS BALD. IN LIEBE. A.F. ${ }^{16}$ Damit aber wird Mein Jenseits eingebettet nicht nur in die postalische Übertragungskette von Dokumenten, die den Roman konstituieren, sondern ebenso in die intime Beziehung von Percy und Feinlein mit ihren latent homoerotischen Zügen. Ich werde im folgenden Abschnitt meines Aufsatzes deshalb zunächst die Nachrichtenketten der einzelnen Formteile nachzeichnen und dabei besonders auf die jeweilige Funktion religiöser Rede in der

${ }^{13}$ Die Unterscheidung eines personalen Prinzips der Verständigung von einer postalischen Medientheorie der Übertragung verdanke ich S. Krämer, Medium, Bote, Übertragung: Kleine Metaphysik der Medialität, Frankfurt 2008.

${ }^{14}$ M. Walser, Mein Jenseits. Novelle, Berlin 2010.

${ }^{15}$ Vgl. die Beiträge in Felder (Hg.), Mein Jenseits: Gespräche über Martin Walsers "Mein Jenseits».

${ }^{16}$ M. Walser, Muttersohn, Reinbek 2011, 357. Zitate im folgenden unter Angabe der Seitenzahl im Haupttext. 
Nachrichtenübermittlung achten. Danach werde ich diskutieren, ob sich aus diesen Nachrichtenketten ein postsäkulares oder ein kunstreligiöses Programm ableiten lässt, um abschließend Rückschlüsse auf die Stellungnahme des Romans zum Spannungsfeld von Rechthaben (müssen) und Leben (können) zu ziehen.

\section{Über religiöse Rede}

"Ewald, ich heiße Percy. Das sagte er, als er die Tür hinter sich zugemacht hatte. Ewald hatte auf sein Klopfen nicht geantwortet." (S. 11) Bereits mit den ersten Sätzen führt der Roman das zentrale Thema der Bedingungen gelingender Kommunikation ein. Fünf Kommunikationssituationen sind konstitutiv für den ersten Romanteil ("Dem Leben zuliebe»): (1) die Erzählungen, die Percy an den in die geschlossene Abteilung des PLK Scherblingen eingewiesenen Ewald Kainz richtet; (2) die öffentlichen Reden Percys im Klostergebäude; (3) die ritualisierten Unterhaltungen Percys mit Augustin Feinlein; (4) die liturgisch organisierten Heilungsrituale, die Percy mit der Patientin Gretel Strauch durchführt; und (5) die Talkshow, in der Percy im letzten Kapitel auftritt und dem Hohn der Öffentlichkeit ausgesetzt wird. Damit stehen »erotische«, d.h. auf Intimität basierende Verständigungen $(1,3,4)$ neben "postalischen « Übertragungen (5; 2 nimmt eine Zwischenstellung ein); einseitige (1,2) neben wechselseitigen Kommunikationen $(3,4,5)$, spontane (1, 2; teilweise auch 3-5) neben ritualisierten (3-5; 2 teilweise) und gelingende $(2,3$, 4) neben misslingenden $(1,5)$ Kommunikationen.

Auffallig ist, dass neben der Jesus-affinen Gestaltung des Protagonisten, dem Setting in einem oberschwäbischen Barockkloster mit seiner plakativen Entgegensetzung von "Religion und Psychiatrie, von Glauben und Neurosen, von Kirche und Pathologie ${ }^{17}$ sowie den zahlreichen expliziten Erwähnungen des Religiösen in der Figurenrede - dass neben diesen bereits oft bemerkten Aspekten das Religiöse eine generative Funktion auch für die Erzählrhetorik übernimmt, denn die fünf skizzierten Kommunikationen, besonders die ersten vier, sind durch religiöse Praktiken, Semantiken und Rituale strukturiert. Dieser strukturgenerative Aspekt steht im Mittelpunkt des Interesses der folgenden Bemerkungen, die sich vor allem auf die in diesem Zusammenhang besonders ergiebigen Kommunikationen (2) und (4) konzentrieren.

${ }^{17}$ Kuschel, `Ohne das Geglaubte wäre die Welt immer noch wüst und leer: Martin Walser über Religion, 75. 
Aber beginnen wir am Anfang: Percy Schlugen, der in Scherblingen als Pfleger ausgebildet wurde, aber seit zwei Jahren ein unstetes Wanderleben durch die oberschwäbischen Pfarrhäuser führt, gilt als begabt darin, Zugang auch zu schwierigen Patienten zu finden - nicht zuletzt deshalb, weil er mit ihnen auf Augenhöhe kommuniziert. Ewald Kainz (über den wir zu diesem Zeitpunkt kaum etwas wissen, denn seine Lebensgeschichte wird erst in dem folgenden Romanteil, »Dieses Leben«, nachgeliefert) zeigt jedoch keinerlei Reaktion, während Percy ihm über mehrere Kapitel die Lebensgeschichte seiner Mutter Fini erzählt - bis er an deren Ende plötzlich aufsteht und seine Schuhe anzieht. Dann jedoch setzt er sich wieder hin und schaut die Wand an. Die knappe Exposition des Romans, die ich oben zitiert habe, enthält somit in nuce bereits das Programm, das der erste Romanteil dann über 146 Seiten, bis zum Ende des 8. Kapitels, ausspinnt. Wie sehr Percy mit seinem Heilungsprogramm des gemeinsamen Schweigens und der anschließenden bekenntnishaften Erzählung scheitert - in welchem Ausmaß diese Kommunikation also gerade kein Verständnis erzeugt - wird aber erst deutlich, wenn die Erzählung Ewald Kainz' im zweiten Teil auf Percys Beichte reagiert. Das tut er nicht mit einer Absolution, sondern mit einer Gegenbeichte, in der sich allerdings keinerlei Bezug auf Percys Beichte findet und die mit Ewalds angekündigtem Selbstmord endet, von dessen Wirkung auf Percy dann im vierten Teil des Romans erzählt wird. Wenn Percy aus dem letzten Gespräch mit Ewald "wie immer" und voller "Wohlgefühl " herausgeht (S. 146), so liegt hier also eine groteske Fehleinschätzung der eigenen Wirkung auf andere vor.

Ganz anders verhält es sich mit Percys öffentlichen Reden (2) und seinen Heilungsritualen mit Gretel Strauch (4). Trugen schon die Besuche bei Ewald erkennbare Züge von Beichtgesprächen, so stehen bei diesen beiden Kommunikationen religiöse Semantiken und Praktiken explizit im Vordergrund. Wiederum ist es instruktiv, die religiösen Motive nicht isoliert zu betrachten, sondern ihrer Einbettung in die Nachrichtenketten des Romans nachzugehen. Der Roman übermittelt zwei Reden Percys im Kloster Scherblingen, berichtet aber auch von mehreren dazwischenliegenden Auftritten in verschiedenen oberschwäbischen Pfarrhäusern. Nicht nur finden Percys Reden an mit der katholischen Kirche verbundenen Orten statt, sie haben auch einen quasi-liturgischen Rahmen. Ihr Bezug zu tradierter Religion ist jedoch ambivalent: das Pfarrhaus ist nicht die Kirche, das Kloster ist ein säkularisiertes Kloster, die Reden finden nach, nicht in der Andacht statt (S. 21) und sie dürfen 
nicht Predigt genannt werden (S. 39f). Ihr ritualisierter Aufbau greift gottesdienstliche und gottesdienstähnliche Elemente auf, ohne eine strikte Formassoziation zuzulassen. Dieser Aufbau soll hier anhand der ersten Rede Percys analysiert werden, die im ersten Kapitel detailliert wiedergeben wird.

Immer beginnen Percys Auftritte damit, dass er auf der Orgel improvisiert, um sich selbst "in Stimmung" zu bringen (S. 22). Mit diesem Präludium ist nicht nur ein gottesdienstliches Ritual angespielt, sondern auch die Frage der richtigen und effektiven Stimmung angesprochen, die nicht nur für den Redner selbst, sondern vor allem für seine persuasive Wirkung auf andere entscheidend ist. Nach diesem eher unspezifischen Einstieg benennt Percy das Motto seines Vortrags: »Liebe Leute. Dem Leben zuliebe. (S. 23) Mit dem "Leben" ist nicht nur Percys tolerantes Programm des Leben und Lebenlassen angesprochen, sondern auch eine emphatische Parteinahme für das Leben im Sinne einer Subjektivität verbunden, die ganz bei sich ist, die spontan und authentisch wirkt - ganz so, wie der Autor Walser es in seinem Wahlstatement der Bundeskanzlerin zugeschrieben hat. Tatsächlich verkörpert die Figur Percy (ebenso wie der zweite Protagonist Augustin Feinlein) nicht nur das in Über Rechtfertigung erläuterte In-die-Welt-gestellt-Sein des gerechtfertigten Menschen, der nicht - wie Feinleins Antagonist Dr. BruderhoferRecht haben muss. Er macht diese Haltung darüber hinaus als eine rhetorische Haltung kenntlich, deren Ziel das Herstellen von Nähe zum Anderen ist: "Jetzt die tolle Hoffnung, er sei anderen am nächsten, wenn er über sich selbst spreche. Jeder sei sich selbst so nah. Da könnten doch alle, die über sich selbst sprechen, einander nah sein." (S. 24) Dem anderen nah sein, indem man von sich selber spricht: das ist nun einerseits in der Tat das Programm, nach dem die Romanstruktur organisiert ist. Denn die setzt sich ja zusammen aus vielen unterschiedlichen Lebensbeichten und Biografien, die durch die Weitergabe von Dokumenten gemäß dem postalischen Prinzip der Nachrichtenübermittlung die Verbindung zwischen den einzelnen Romanteilen stiften. Gleichzeitig ist jedoch durchaus fraglich, inwiefern diese formale "Nähe" auch in der Lage ist, Intimität zwischen den Figuren zu stiften. Percys beziehungsloses Leben und seine völlige Fehleinschätzung der Jollynecks sprechen ebenso gegen die Persuasivität dieses Programms wie der Selbstmord Ewald Kainz'. Darüber hinaus kann von hier aus auch die Persuasivität des Romans selbst in Frage gestellt werden: Will nicht auch der Autor Walser uns, seinen Lesern, nahe sein oder kommen, indem er ganz bei sich ist? Denn der Roman enthält, als Figuren- 
rede verfremdet, zahlreiche Überlegungen zu Religion und Poetik, die sich fast wortidentisch auch in nicht-fiktionalen Texten Walsers finden und offenbar als eine Art Botschaft ernst genommen werden sollen. Insbesondere Feinlein fungiert in Muttersohn als ein Sprachrohr für Walser-Sätze - gerade dann, wenn vom als-ob des Glaubens, einem der Kernthemen von Über Rechtfertigung, die Rede ist. ${ }^{18}$ Auch Walsers Satz aus einem Interview mit Karl-Josef Kuschel: „Gäbe es Gott, dann gäbe es kein Wort dafür«, wird im Roman Feinlein in den Mund gelegt (S. 331). ${ }^{19}$ Wenn aber der Roman weiß, dass das Bei-sich-sein nicht Verständigung oder gar Überzeugung herstellen kann: wie steht es dann um seine eigene Persuasivität? Dem Autor Walser scheint das Problem jedenfalls nicht bewusst zu sein, nimmt man sein Wahlstatement beim Wort (oder ist es nur Klamauk?).

Hinzu kommt ein zweites. Denn der Anspruch einer Wirkung durch Subjektivität steht in einem spannungsvollen Verhältnis zu der Art, in der Percys Reden, aber auch die Äußerungen und Schriften Augustin Feinleins, religiöse Semantiken einspielen - speziell die christliche Tradition mit ihrem weitaus radikaleren Projekt der selbstentäußernden Liebe. Der Spagat gelingt im Roman deshalb, weil Percy sich zwar einerseits explizit auf das Reich Gottes beruft, den Ausdruck dann aber zu einer ungreifbaren Stimmung degradiert, über die sich nicht spezifisch sprechen lasse. »Er, Percy, gebe zu, dass das ein schöner Ausdruck sei: Reich Gottes. Das hat was. Aber man weiß nicht, was es hat. Auf ihn wirke es wie Musik oder wie eine Droge. Er könne aber zu anderen nicht über Musik oder Drogen sprechen." (S. 24) Auch an anderen Stellen des Romans sollen theologische Begriffe eine affektive Aufladung kommunizieren, die von jeglicher Bedeutung abgeschnitten wird. Damit wird eine religiöse Sprache reproduziert, die jeder theologischer Referenz entbehrt und statt dessen dazu dient, ein ästhetisches Erleben zu produzieren - so wenn Augustin Feinlein in Mein Jenseits schreibt: "Woher die

\footnotetext{
${ }^{18}$ Z.B. schreibt Feinlein in "Mein Jenseits«: "Wenn es den Himmel gäbe, könnten wir nicht daran glauben. Erst wenn uns auffällt, dass wir daran glauben, merken wir, dass wir nicht daran glauben. Dieses Nichtglauben unterscheidet sich kein bisschen vom Glauben. Das ist EINE Art von Gefühl oder Existenz. Immer unterschieden vom Wissen." (329)

${ }^{19}$ Im Interview sagt Walser:»Wenn wir Gott hätten, hätten wir kein Wort dafür.« K.-J. Kuschel, Das wäre meine Religion: Gespräch mit Martin Walser, in: Ders. (Hg.), Weil wir uns auf dieser Erde nicht ganz zu Hause fühlen". 12 Schriftsteller über Religion und Literatur, München 1985, 146. Zu Recht schließt Kuschel, Walser schaffe sich in seinem Werk "Ersatzfiguren, mit deren Hilfe er ausprobiert, wie die Rede von Gott 'funktionieren « kann und wie andere darauf reagieren «. > Ohne das Geglaubte wäre die Welt immer noch wüst und leer: Martin Walser über Religion, 74.
} 
[Caravaggios "Madonna dei Pellegrini«, SH] ihren Jesus hat, wagt man nicht zu denken. Allein die Schönheit zählt. Das Jenseits muss schön sein. Sonst kannst du es gleich vergessen." (S. 297) Es handelt sich hierbei um eine Form "religiöser Rede", die Bruno Latour so beschreibt: " $\operatorname{Er}$ [damit meint Latour sich selber, $\mathrm{SH}$ ] will bloß dem religiösen Ausdruck wieder Bewegungsfreiheit verschaffen, diesem so einzigartigen Brauch, der im Lauf der Geschichte Wort und Sprache gewann und der ihm heute so entsetzlich gehemmt vorkommt. $\mathbb{1}^{20}$

Auch Walser geht es nicht um die Existenz Gottes, sondern darum, wie über Ihn geredet wird. In dem Essay "Umgang mit Unsäglichem", der in Auseinandersetzung mit den theologischen Beiträgen zu Mein Jenseits entstand, schreibt er: "Gott wäre natürlich prima. Aber er könnte, wenn es ihn gäbe, nicht deutlicher sein, als er durch seine Abwesenheit ist. So ist er das Wort für alles, was mir fehlt. $\|^{21}$ Die hier vorgenommene Ersetzung einer genuinen Transzendenzbeziehung durch das Ästhetische findet ihren konsequenten Abschluss in dem Gedicht, mit dem Percy seine Rede beendet (S. 26):

"Nun habt ihr gut geschlafen

und wünscht euch fort von hier

zu Kräutern und zu Schafen, und zwei mal zwei bleibt vier.

Einziehen, schließen, falten, eben leben, keine Steigerung, den Fall nicht nähren, nur Schnüre entwirren und sorgsam enden."

Kräuter, Schafe und ein einfaches Leben: sicherlich ist Percys Rede mit einem Augenzwinkern zu lesen; dennoch kann wohl mit Fug und Recht (nicht nur, aber auch auf der Basis der eigenen Leseerfahrung) bezweifelt werden, dass das biedere Plädoyer für die oberschwäbische Öko-Idylle dazu führt, dass die Zuhörer eine "mich übersteigende Fähigkeit, dass ich etwas schön finden kann" empfinden - nach Walser ist das der Erlösungsersatz, den das Ästhetische bietet. ${ }^{22}$ Ist es doch vor allem Percy selbst, der den Saal gerührt

\footnotetext{
${ }^{20}$ B. Latour, Jubilieren: Über religiöse Rede, übers. v. A. Russer, Berlin 2011, 8 f.

${ }^{21}$ M. Walser, Umgang mit Unsäglichem, in: J.-H. Tück (Hg.), Was fehlt, wenn Gott fehlt? Martin Walser über Rechtfertigung - theologische Erwiderungen, Freiburg 2013,11-16, 15.

22 „Solange du etwas schön findest, bist du erlöst. Erlöst von dir. Und nachher, als Gewesenes, bleibt Sehnsucht." Beide Zitate in: ebd.
} 
von der eigenen Wirkung verlässt - und zwar absichtlich so, »dass er niemandem begegnen musste.« (S. 26)

Werfen wir vor diesem Hintergrund einen Blick auf die Kommunikation (4), also Percy Schlugens Heilungsrituale mit Gretel Strauch, so zeigt sich hier eine vergleichbare egozentrische Konzentration und das identische Sinnentleerungsprogramm im Umgang mit religiösen Semantiken und Ritualen. Percys sogenannte "Schlafsackbehandlung « besteht nämlich darin, mit der Patientin Gretel bis zur Erschöpfung barfuß und Hand in Hand an den Wänden einer ehemaligen Klosterscheune entlang $z u$ gehen und dabei auswendig gelernte Textfragmente von Swedenborg, Seuse und Jakob Böhme zu rezitieren, bevor sie erschöpft in die Schlafsäcke sinken. Die Bricolage von Semantiken, Anspielungen und Diskursformen in dieser religiösen Rede reproduziert die Form der ambivalenten, hermetischen und subversiven Zitation, wie sie - bei offensichtlichen qualitativen Unterschieden - aus der Lyrik Hölderlins und Hardenbergs bekannt ist. ${ }^{23}$ Doch dient das Aufrufen hermetischer Traditionen im Grenzbereich von Christentum und Spiritismus nicht einer Auseinandersetzung mit deren Inhalten; es geht auch nicht darum, dass die Texte geglaubt werden, sondern lediglich um ihre Wirkung: »Wie es ihnen dann in uns ergeht, wird sich zeigen.» (S. 152) Auch die Pragmatik des Rituals spielt zwar Traditionen der Pilgerschaft und der Wallfahrt ein, doch führt der Weg Percys und Gretels tatsächlich nirgendwohin, und ob eine Heilung stattfindet, bleibt ungewiss. Denn wiederum wird der erfolgreiche Empfang dieser Kommunikation nicht bei der Rezipientin, bei Gretel Strauch, sondern nur - bei Percy selbst überprüft: er ist es, der sich einbildet, »ihre Schritte seien sicherer als vor zwei Tagen. Und ihr Blick, als sie Non sum sagte, war hell.» (S. 153) Ob Gretel Strauch tatsächlich keine Stimmen mehr hört: Percy scheint es nicht zu interessieren, und der Roman lässt es uns nicht wissen.

\section{Über Kunstreligion}

Nicht nur Stimmungen, Bekenntnisse, Dokumente oder Dinge wie die Heiligblutreliquie oder die Asche Ewald Kainz' werden in Muttersohn übertragen (oder eben gerade nicht): auch die strukturelle Rahmung dieser gelingenden oder scheiternden Übermittlungen lebt von einem Übertragungsprozess, der als symbolischer Reflex

${ }^{23}$ Vgl. J.A. Steiger, Die Sehnsucht nach der Nacht: Frühromantik und christlicher Glaube bei Novalis (1772-1801), Heidelberg 2003. 
der romaninternen Auseinandersetzung mit kunstreligiösen und postsäkularen Programmen gelesen werden muss. Denn beide Rahmenteile spielen in säkularisierten Klöstern - religiösen Orten, die in der Moderne nicht-religiös genutzt werden. Dabei kommt der veränderten Situation im vierten Teil ("Fortleben«) eine besondere Bedeutung zu, gerade in Bezug auf das Verhältnis von postsäkularen und kunstreligiösen Argumentationsfiguren.

Mit der Wahl zweier ehemaliger Klöster - Scherblingen und Rheinau - als Orten der Rahmenhandlung ist eben nicht nur die katholische Tradition in Oberschwaben und am Oberrhein aufgerufen, sondern auch die Frage gestellt, wie nach dem Abreißen dieser Tradition in der Säkularisierung mit den verbleibenden Traditionsbeständen umgegangen werden kann und darf. Nicht zufällig fungiert das Kloster in der Literatur um 1800, im historischen Kontext der Säkularisation also, als ein literarischer Imaginationsort, an dem die Transformation von Religion in Kunstreligion in ihrer ganzen Ambivalenz reflektiert wird. ${ }^{24}$ Diese Transformation bildet nun auch das Strukturgerüst von Muttersohn. Sie wird in zwei Schritten vollzogen. Der erste ist der Übergang vom Kloster zur Klinik in Scherblingen. Signifikant ist, dass dieser Übergang nicht vollständig vollzogen wird: auch nach dem Neubau moderner Klinikgebäude wird das alte Kloster weiter genutzt. Augustin Feinlein unterhält dort ein Arbeitszimmer, auch Percys Reden und ein Filmabend mit Urlaubsfilmen des ärztlichen Direktors Dr. Bruderhofer finden im alten Klostergebäude statt. Grob kann man sagen, dass die Medizin umgezogen ist, die Kunst jedoch noch im Kloster wohnt. Eine Verbindung zwischen beiden Sphären stiftet Augustin Feinlein: er ist der Chef des PLK Scherblingen, zugleich aber Nachfahre eines Scherblinger Abtes und unterhält Räume in beiden Gebäuden. In diesem ambivalenten Zwischen präsentiert der Roman Scherblingen als einen utopischen Ort der Kunst und der Heilung: Feinlein und Percy entwickeln hier Heilungsrituale im Gegensatz zur von Dr. Bruderhofer praktizierten schulmedizinischen Behandlung mit Psychopharmaka; im Rechtfertigungskomplex Martin Walsers nimmt

\footnotetext{
${ }^{24}$ Man denke nur E.T.A. Hoffmanns Elixiere des Teufels, deren Held - der Klosterbruder Medardus - durch seine Redekünste einerseits, das Erbe eines teuflischen Malers andererseits ins Verderben gestürzt wird. Zu Kunstreligion vgl. im übrigen B. Auerochs, Die Entstehung der Kunstreligion, Palaestra 323, Göttingen 2006; ders., Das Bedürfnis der Sinnlichkeit. Möglichkeiten funktionaler Äquivalenz von Religion und Poesie im 18. Jahrhundert, in: A. Meier/A.Costazza/G. Laudin (Hg.), Kunstreligion. Bd. 1: Der Ursprung des Konzepts um 1800, Berlin/New York 2011, 29-44; $\mathrm{H}$. Detering, Was ist Kunstreligion? Systematische und historische Bemerkungen, in: Meier/Costazza/Laudin (Hg.), Kunstreligion, 11-27.
} 
Bruderhofer deshalb die Stellung des Rechthabers im Gegensatz zu den auf sympathische Weise unterlegenen, aber gerechtfertigten Figuren Percy und Feinlein ein. Zudem ist Scherblingen nicht nur ein wunderschönes Barockkloster, sondern auch Ort der Produktion zweier bemerkenswerter literarischer Werke: erstens des "Scherblinger Schweigens" aus der Feder Augustin Feinleins, eines Textes unklarer Gattungszugehörigkeit, das aus Feinleins "Empfindungsgeschichte« stammt und nach Meinung Dr. Bruderhofers deshalb "kein Fach- und Sachbuch" sein kann (S. 38). Dass "Scherblinger Schweigen" auch der Name einer Behandlungsmethode Percys ist, die er anfänglich mit Ewald Kainz praktiziert (nämlich stundenlanges Anschweigen), schließt die Utopie der Heilung plakativ mit der Kunstutopie kurz. In diesen Kurzschluss gehört auch - zweitens - die von dem Patienten Innozenz (eigentlich Horst-Jürgen Storch) herausgegebene "Scherblinger Anthologie» mit dem Titel „OFENKÜCHE« (S. 101). Dabei handelt es sich um ein Gegenbuch $\mathrm{zu}$ einem Literaturbetrieb, in dem das Abseitige aus dem Programm verdrängt wird: die Scherblinger Anthologie soll alles enthalten, was anderswo nicht gedruckt wird. Damit stellt sie das literarische Pendant zum PLK Scherblingen dar, das selbst ein Repositorium für verdrängtes Wissen, überflüssige Dinge und unerwünschte Menschen bildet, und sie formuliert aus diesem Widerspruch ein Programm der Öffnung für das vermeintlich Unwichtige, aber auch Arkane und Esoterische. ${ }^{25}$

Die hier bereits sich andeutende Verwandlung von Klinik in Kunst wird in einem zweiten Schritt mit dem Übergang vom dritten zum vierten Romanteil vollzogen: denn das Kloster Rheinau, in das Percy und Feinlein nach dem Skandal um Feinleins Reliquiendiebstahl umziehen, ist nicht nur wie Scherblingen ein säkularisiertes Kloster, das tatsächlich bis 2000 als psychiatrische Klinik genutzt wurde, es soll nun explizit $\mathrm{zu}$ einem Kulturzentrum ausgebaut werden. ${ }^{26}$ In einer "Akademie für Unvollendete« sollen alle Besucher unabhängig

\footnotetext{
${ }^{25}$ Die Scherblinger Anthologie soll nicht nur das Abgelehnte und Unerwünschte "unauffindbar für die Spürhunde des Zeitgeists" machen (S. 101), sondern vor allem auch solche Texte enthalten, die einen religiösen Wahn dokumentieren. Beispielsweise schreibt ein Hans Sperber an Innozenz: "Wenn es aber einen Gott gibt, so gibt es zu allen Zeiten nur eine Sprache, in der man zu diesem Gott redet, wenn man von ihm angerufen wird. Darum kann ich nicht zustimmen, wenn mir gesagt wird, dass in meine Dichtungen nichts eingegangen sei, was der Mitteilung wert ist. Ich bin angerufen worden von Gott." (S. 103)

${ }^{26}$ Tatsächlich soll das momentan leerstehende Kloster Rheinau bis 2014 zu einer "Schweizer Musikinsel Rheinau" ausgebaut werden. Vgl. die Website des Projekts: <http://www.inselrheinau.ch/> (eingesehen am 16.9. 2013).
} 
von Ausbildung und Begabung musizieren dürfen - es gibt dann nur noch Produzenten von Kunst, keine Rezipienten mehr. Gleichzeitig radikalisiert das Rheinauer Programm der universalen Inklusion die in Scherblingen entwickelte, in der Romantik verwurzelte Poetik das absoluten Buches: durch die Schreddermaschine "Oblomov" wird es möglich, tatsächlich alle Einsendungen zu veröffentlichen, ohne in Material- und Raumnot zu gelangen - denn alles Geschriebene wird sofort wieder vernichtet. ${ }^{27}$

Auch hier bietet sich eine Parallele zur Form des Romans Muttersohn an, der ja tatsächlich das bewahrt und weitergibt, von dem er erzählt: die Schwundstufe des Religiösen ebenso wie den nicht literaturfäigen Text, die Bekenntnisse der Figuren ebenso wie ihre Entblößung im TV-Entertainment. Handelt es sich bei Muttersohn also um eine späte Verwirklichung des romantischen Programms einer progressiven Universalpoesie, die überall zugleich Poesie und Poesie der Poesie ist? Ja und nein. Sicherlich sind die Bezüge auf die romantische Poetik im Religions- wie im Literaturdiskurs des Romans unübersehbar. Doch erreichen Walser und seine Figuren keineswegs den Komplexitätsgrad der Wackenroder/Tieck'schen Herzensergießungen eines kunstliebenden Klosterbruders. Wenn Walser seinen Augustin Feinlein schreiben lässt: „Die Caravaggio-Madonna hat es gegeben. Sie ist mein Jenseits. An sie zu glauben ist einfach. Durch sie wird die Welt schöner, als sie ist." (S. 327) - so bleibt dies eine ziemlich substanzlose Behauptung (während bei Wackenroder Vision, Imagination, Produktion und Rezeption von "Raffaels Erscheinung « in ein komplexes Wechselspiel gesetzt werden). Auch da, wo sie rernsthaft - sprich: postsäkular und nicht kunstreligiös - erscheinen, werden die religiösen Stellungnahmen der Figuren immer wieder durch die narrative Einbettung gebrochen, und zwar so, dass sie sich in klamaukartige Motive im Dienste der Leserbelustigung verwandeln (wenn man so etwas denn lustig findet). Das betrifft insbesondere die von den theologischen Lesern vielzitierten Sätze Augustin Feinleins über den Glauben. Sie fallen im Kontext seiner Rechtfertigung für den Diebstahl der Scherblinger Heiligblut-Reliquie. Feinlein gibt folgendes zu Protokoll: Man darf das Spektakel

\footnotetext{
${ }^{27}$ Zum frühromantischen Projekt des absoluten oder "vollständige[n] Buch[es] « vgl. Novalis: Schriften. Die Werke Friedrich von Hardenbergs. Nach den Handschriften ergänzte, erweiterte und verbesserte Auflage, hg. v. P. Kluckhohn/R. Samuel in Zusammenarbeit mit H.J. Mähl u. G. Schulz, 2. Aufl., Stuttgart 1960ff. Bd. III, 273:185. S. auch Novalis' Brief an Friedrich Schlegel vom 7.11.1798 (N IV, 262f) und Schlegels Antwort (N IV, 506f). Zur Umsetzung dieses Programms im Werk Clemens Brentanos vgl. meine Dissertation:Androgyne Autorschaft. Poesie und Geschlecht im Prosawerk Clemens Brentanos, Tübingen 2004.
} 
[gemeint ist der Scherblinger Blutritt, der auf dem realen Weingartener Blutritt basiert, $\mathrm{SH}$ ] wegen der reitenden Bauern rührend und schön finden. Alles, was die Kirche dazu zu bieten hat, ist eine Verlegenheit. Keiner dieser Bischöfe, keiner dieser geistlichen Herren glaubt daran, dass das im Bergkristall wirklich ein paar Blutstropfen Christi seien. Sie tun aber so, als glaubten sie." (S. 348) Doch geht es Feinlein bei seinem Diebstahl gar nicht um eine Rehabilitation des Wunderglaubens, sondern es handelt sich um eine Affektreaktion darauf, dass der Konkurrent Dr. Bruderhofer ihm beim Silvestertanz die von Feinlein angebetete Sekretärin Luzia Meyer-Horch ausgespannt hat. Indem aber Feinleins Plädoyer für den Reliquienglauben als verzweifelte Handlung eines unglücklich Liebenden dargestellt wird, wird aus seinem "Jenseits", Hildegard Wustmans hat darauf hingewiesen, ein "Nullsummenspiel« diesseitiger Hoffnungen, Wünsche und Bedürfnisse. ${ }^{28}$ Auch wegen dieser narrativen Einbettung dürfen die Feinlein-Sätze nicht mit ähnlich lautenden Walser-Sätzen verwechselt werden. ${ }^{29}$

\section{Fazit: Über gerechtfertigtes Schreiben}

Von Kloster über Klinik zu Kunst als Klamauk also: wie ist angesichts dieses Transformationspfades Walsers Plädoyer für das Leben (können) als rhetorisch-poetisches Prinzip zu bewerten? Wie rechtfertigen sich seine Figuren, und welche Position nimmt der Roman zur Rechtfertigungsfrage ein? Führen wir die Figurenkonstellation auf den von Walser postulierten anthropologischen Grundwiderspruch von Rechthaben (müssen) und Leben (können) zurück, so steht Dr. Bruderhofer mit seiner Berufung auf fachspezifische Diskursregeln, auf das Erlernte und gesellschaftlich Akzeptierte für eine Haltung des Rechthaben (müssen)s. Percy und Augustin Feinlein mit ihrem Dilettantismus hingegen verkörpern die echte, authentische und einfache Rhetorik des Leben (können)s. Plastisch wird diese Haltung in Percys öffentlichen Reden im Kloster Scherblingen vorgeführt. Allein: auch wenn Walser von sich behauptet, er lasse sich durch die Rhetorik des Leben (können)s überzeugen - Percy überzeugt mit seinen Reden niemanden. Ewald Kainz begeht nach

\footnotetext{
${ }^{28}$ H.Wustmans, Mein Jenseits - nicht mehr als ein Nullsummenspiel, in: Felder (Hg.), Mein Jenseits: Gespräche über Martin Walsers »Mein Jenseits«, 30-35.

${ }^{29}$ Wie das etwa der Pastoraltheologe Erich Garhammer tut, wenn er Figur und Autor einfach gleichsetzt: E. Garhammer: /Zuflucht Sprache $<-Z$ u den Confessiones des Dr. Augustin Feinlein alias Martin Walser, in: Felder (Hg.), Mein Jenseits, 62-71.
} 
Percys Behandlung Selbstmord, die öffentlichen Reden und das Heilungsritual mit Gretel Strauch haben eine Wirkung vor allem auf ihn selbst, und sein Auftritt in der Talkshow endet im hysterischen Gelächter einer verständnislosen Mediengesellschaft. Auch Augustin Feinleins Bekenntnisschrift Mein Jenseits zerfasert im inkohärenten Gestammel eines liebestollen Geisteskranken.

In dem Essay Umgang mit Unsäglichem formuliert Walser das Ziel seiner Poetik, andere zu berühren und umgekehrt berührt zu werden. Das sei aber unkalkuliert, fast schon kontingent: "Ich möchte mich unwillkürlich äußern und es nicht wissen müssen, wie und wo es einen anderen berührt. ${ }^{30}$ Die hier behauptete Unmittelbarkeit und Planlosigkeit ist jedoch eine Geste. Tatsächlich ist der Roman Muttersohn, wie ich gezeigt habe, äußerst planvoll durchstrukturiert. Und das in dieser Struktur entwickelte Plädoyer für eine Rechtfertigung in der Kunst, das zunächst nach Toleranz für eine ganz einfache Form der Unmittelbarkeit in einem beinahe erotischen Verhältnis intimer Verständigung klingt, erweist sich bei näherem Hinsehen als eine Form egozentrischer Unverbindlichkeit. Das weiß auch der Roman, wenn er gerade die Figuren, die eben nicht schon Teil des in Rheinau sich etablierenden Kunstbetriebs sind - Ewald Kainz, Augustin Feinlein und Percy Schlugen - sterben lässt. Letztlich ist die Kunst, auch in Rheinau, eben doch keine Kunst für alle, sondern für Experten des Kulturbetriebs wie Ewalds Witwe, die Chordirigentin Elsa Frommknecht, die die Leitung der Akademie übernimmt. Das Expertentum aber ist, nach Walsers Logik, die Haltung des Rechthabens - nicht des freien und interesselosen Gerechtfertigtseins. Ironisch und fast schon hellsichtig mutet es vor diesem Hintergrund an, dass Percy am Ende des Romans von den Jollynecks ermordet wird, deren Symbol der erhobene Mittelfinger ist: die Geste, mit der Peer Steinbrück kurz vor der Bundestagswahl auf der Titelseite des Süddeutsche-Magazins einen Sturm im Blätterwald auslöste. ${ }^{31}$

— PD Dr. Silke Horstkotte ist Privatdozentin für neuere deutsche Literatur am Institut für Germanistik der Universität Leipzig.

${ }^{30}$ Walser, Umgang mit Unsäglichem, 11.

${ }^{31}$ Süddeutsche-Magazin, 14.9. 2013. 